\section{The orienting reflex and amount and direction of conceptual novelty*}

\author{
R. M. YAREMKO, San Diego State College, San Diego, Calif. 92115 \\ and \\ KENNETH KELEMAN, University of Utah, Salt Lake City, Utah 84112
}

The galvanic skin response component of the orienting reflex (OR) was recorded for four groups of 11 Ss. They viewed seven numeric stimuli presented sequentially, then a test stimulus (TS) which was out of sequence by \pm 3 or \pm 15 units, followed by a resumption of the sequence. It was found that ORs to the TS were a function of the amount of disparity between that TS and the "expected" stimulus and that direction of that disparity was not an effective manipulation. There were no such systematic changes in OR recovery to the following in-sequence stimulus. The results were related to previous research using both numeric and simple sensory stimuli and to Sokolov's account of the OR.

A number of recent studies have examined Sokolov's (1963) characterization of the orienting reflex (OR). In general, this research has shown that one very important determinant of OR magnitude is the amount of discrepancy, on one or more dimensions, between some ORevoking stimulus and ambient stimulation, or some "expected" stimulus. Direction of stimulus change usually influences OR magnitude only to the extent that dynamogenic factors may be introduced by this manipulation (e.g., Kimmel, 1960; Yaremko, 1969).

While many of these studies employed neutral, purely "sensory" stimuli such as lights or tones, a few have studied the OR to changes in the "conceptual" dimension provided by presenting a predictable series of numbers followed by an out-of-sequence test stimulus (TS). Unger (1964) showed that the vasomotor component of the $O R$ habituated to a series of aurally received numbers and was reinstated by the TS. Zimny, Pawlick, \& Saur (1969) found no differences in habituation rates to predictable and unpredictable series of numbers but did show dishabituation of the galvanic skin response (GSR) component of the OR to a TS interpolated in the predictable series.

Yaremko, Blair, \& Leckart (1970) investigated the effects of amount of change in numeric units between an expected stimulus and the TS and the direction (positive or negative) of that change on the GSR-OR. The numerals $10-13$ were presented visually, and the

* This research was supported in part by a grant to the first author from the San Diego State College Foundation Address requests for reprints to $R$. M. Yaremko, Department of Psychology, San Diego State College, San Diego, Calif. 92115 . number 20 (which $S$ presumably expected) was replaced by $9,19,21$, or 31 . Thus, the novel TS took on values of \pm 1 or \pm 11 units. Although there was some tendency for negative direction TSs to evoke smaller ORs, response magnitude was primarily a function of the amount of change in the TS.

One difficulty with that study, however, was that TS 19, although conceptually novel, was physically familiar, rendering its status as an "appropriate" TS somewhat ambiguous. A similar argument could be raised regarding TS 9. As the authors acknowledged, any attempt to compare these findings with those of studies employing simple, physical stimulus dimensions must be done cautiously. However, the implications of that study for extending at least some of the parameters of the OR into the domain of conceptual dimensions most certainly warrants further investigation.

The present experiment was designed to allow a novel, negative direction TS to be interpolated back into the habituation series without having to use a previously delivered number. As with other research, the minor procedural changes adopted for this study would permit greater generalization of the combined outcomes of both studies by weakening the argument that the results were uniquely associated with the operations and stimuli used in either study.

SUBJECTS AND APPARATUS

Twenty-eight female and 16 male undergraduates at San Diego State College volunteered in order to meet a class requirement. Data were collected from the $S$ seated in an IAC Model 401-A chamber containing a cushioned chair. The GSR was picked up from the palm and volar surface of $\mathrm{S}$ 's right forearm by 14-mm-diam zinc-zinc sulfate electrodes in Lucite cups filled with Redux electrode jelly. The response was amplified and recorded by a Grass Model 5-D polygraph containing a Model 5P1 low-level dc preamplifier. Paper speed was $1.5 \mathrm{~mm} / \mathrm{sec}$. Numbers were projected on a translucent Plexiglas screen $(35 \times 55 \mathrm{~cm})$ mounted on the chamber window in front of $\mathrm{S}$ by a Kodak Model 850 projector. A light-tight tunnel extended from the projector lens to the screen. The numbers were black on white and approximately $20 \mathrm{~cm}$ high when projected. The distance between $S$ and the screen was approximately $1.0 \mathrm{~m}$. DESIGN AND PROCEDURE

A 2 by 2 factorial design placed amount of change of the TS orthogonal to the direction of that change. Upon reporting to the laboratory, the $S$ was assigned randomly to one of the four experimental conditions, with the exception that each group had $n$ members before any group had $n+1$ members. The S's hand was cleaned with acetone and the electrodes were attached. At this time, he was told informally that the experiment was designed to study simple physiological responses to external stimulation. $\mathrm{He}$ was cautioned to remain alert, avoid excessive movement, and to view whatever appeared on the screen. These instructions were repeated through an intercom after $\mathrm{S}$ was seated in the chamber. Following a 5-min initial rest period, the numbers 10,12 , $14, \ldots, 22$ were delivered serially to $S$. On the eighth trial (when 24 was expected), TS $9,21,27$, or 39 was delivered. Thus, the TS was either \pm 3 or \pm 15 units out of sequence. On the next trial, 24 was presented and the series terminated. Stimulus duration was always $10 \mathrm{sec}$, and the intertrial interval varied between 30 and $60 \mathrm{sec}$, with a mean of $45 \mathrm{sec}$.

\section{RESULTS}

An OR was defined as the first GSR to occur within 1.5 to $6.0 \mathrm{sec}$ of stimulus onset. The response was transformed to log conductance units by the formula: log (resistance before/resistance after) $x$ 1,000. The measure of orienting to the TS was S's response to the TS minus his mean response amplitude to the last three in-sequence stimuli. These data are presented in Table 1. The tabled values strongly suggest that only the amount of change manipulation was effective in modifying orienting behavior. Analysis of variance indicated that the main effect for amount of change was significant $(\mathrm{p}<.025)$ and that neither the direction of change nor their interaction were significant $(F<1.0$ for each). Supplemental analyses revealed that within each direction of 
Table 1

Mean GSR Magnitudes $(\triangle \log C \times 1000)$ for Each Direction and Amount of Change Condition

\begin{tabular}{lcc} 
Direction & \multicolumn{2}{c}{ Amount of Change } \\
\cline { 2 - 3 } of Change & 3 & 15 \\
\hline Positive & 39.2 & 58.4 \\
Negative & 41.1 & 58.5 \\
\hline
\end{tabular}

change condition, the difference between large and small change groups approached significance $(p<.10$ for each). Inspection of the data from the ninth trial (when the series was resumed) showed that while some Ss displayed observable $\mathrm{OR}$ recovery, no reliable trends were uncovered.

\section{DISCUSSION}

The test trial data quite clearly show that, irrespective of the direction in which a novel stimulus departs from an expected one, the OR evoked is a function of the amount of discrepancy provided by the TS. The results, therefore, confirm the findings of Yaremko et al (1970) and are consistent with Sokolov's predictions and previous $O R$ research with simple sensory stimuli. The present findings also extend those of Unger (1964) and Zimny et al (1969).
Not unexpected was the fact that OR recovery to the resumed series did not correspond to the behavior elicited by the TS. This phenomenon does seem to occur with simple sensory stimuli (e.g., Zimny \& Schwabe, 1965) and is part of Sokolov's characterization of the OR. It was not, however, clearly present in the Zimny et al (1969) or Yaremko et al (1970) studies. Thus, it appears that this aspect of orienting behavior may not transcend the level of simple sensory stimuli.

The present results also provide commentary on an alternative explanation (Ely, 1971) of some of our previous results. Ely maintained that ORs to the TSs 9 and 19 were smaller because of the conceptual proximity of these stimuli to elements of the already exposed series. That is, 9 was conceptually closer to the series 10-19 than was 31. The present results seem to offer little support for this view, since mean ORs for positive and negative direction TSs were nearly equivalent.

Be that as it may, this and previous studies on habituation to numeric stimuli certainly suggest that at least some of the parametric properties of the OR are not peculiar to the domain of simple sensory stimulus manipulations.

$$
\text { REFERENCES }
$$

ELY, D. J. A comment on the Yaremko, Blair, \& Leckart report: The orienting reflex to changes in a conceptual stimulus dimension. Psychonomic Science, 1971. $23,414$.

KIMMEL. H. D. The relationship between direction and amount of stimulus change and amount of perceptual disparity response. Journal of Experimental Psy chology, 1960, 59, 68-72.

SOKOLOV, Ye. N. Perception and the conditioned reflex. New York: Pergamon Press, 1963.

UNGER, S. M. Habituation of the vasoconstrictive orienting reaction. Journal of Experimental Psychology, $1964,67,11-18$

YARFMKO, R. M. Effects of direction and amount of change in stimulus intensity on magnitude of the orienting reflex and frequency of signal detection. Unpublished PhD dissertation. Ohio University, 1969.

YAREMKO, R. M., BLAIR, M. W., \& LECKART, B. T. The orienting reflex to changes in a conceptual stimulus dimension. Psychonomic Science, 1970, 21, 115-116.

ZIMNY, G. H., PAWLICK, G. F., \& SAUR, D. P. Effects of stimulus order and novelty on orienting responses. Psychoph y siology, 1969, 6, 166-173.

ZIMNY, G. H., \& SCHWABE, L. W Stimulus change and habituation of the orienting reflex. Psychophysiology, 1965. 2, 103-115. 